\title{
Conceptual Design of a Compact Positron Tomograph for Prostate Imaging
}

\author{
J. S. Huber, Member, IEEE, S. E. Derenzo, Fellow, IEEE, J. Qi, Member, IEEE, W. W. Moses, \\ Senior Member, IEEE, R. H. Huesman, Senior Member, IEEE, and T. F. Budinger, Member, IEEE
}

\begin{abstract}
We present a conceptual design of a compact positron tomograph for prostate imaging using a pair of external curved detector banks, one placed above and one below the patient. The lower detector bank is fixed below the patient bed, and the top bank adjusts vertically for maximum sensitivity and patient access. Each bank is composed of 40 conventional block detectors, forming two arcs $(44 \mathrm{~cm}$ minor, $60 \mathrm{~cm}$ major axis) that are tilted to minimize attenuation and positioned as close as possible to the patient to improve sensitivity. The individual detectors are angled to point towards the prostate to minimize resolution degradation in that region. Inter-plane septa extend $5 \mathrm{~cm}$ beyond the scintillator crystals to reduce random and scatter backgrounds. A patient is not fully encircled by detector rings in order to minimize cost, causing incomplete sampling due to the side gaps. Monte Carlo simulation (including randoms and scatter) demonstrates the feasibility of detecting and differentiating partial and whole prostate tumors with a tumor to background ratio of $2: 1$, utilizing the number of events that should be achievable with a 6-minute scan after a $10 \mathrm{mCi}$ injection (e.g., carbon-11 choline or fluorine-18 fluorocholine).
\end{abstract}

\section{INTRODUCTION}

$\mathrm{W}$ E present a conceptual design of a compact positron tomograph optimized to image the prostate. This instrument images radiopharmaceuticals that specifically localize in the prostate to confirm the presence, absence or progression of disease. It will have approximately 4 times less detectors than a conventional whole-body positron emission tomograph (PET), which will reduce the cost and increase clinical availability.

Prostate cancer has a prevalence and diagnostic rate similar to breast cancer, with 360,000 new cases diagnosed each year and 2 million men affected by the disease. Four percent of men over 50 will have clinically significant disease [1]. Currently, prostate cancer suspicion is typically based on an elevated prostate-specific antigen (PSA) level or a suspicious node found during a digital rectal exam. Serum PSA values do not always correlate well with clinical diagnosis or outcomes [2-4]. Palpation is subjective, insensitive and inexact; more than half of all cancers detected today are not

Manuscript received November 3, 2000. This work was supported by the Director, Office of Energy Research, Office of Health and Environmental Research, Medical Applications and Biophysical Research Division of the U.S. Department of Energy under contract No. DE-AC03$76 \mathrm{SF} 00098$.

J. S. Huber, S. E. Derenzo, J. Qi, W. W. Moses, R. H. Huesman and T. F. Budinger are with the Lawrence Berkeley National Laboratory, Mailstop 55-121, 1 Cyclotron Road, Berkeley, CA 94720 USA (telephone: 510-4866445, e-mail: jshuber@lbl.gov). palpable. Treatment decision is based mainly on biopsy confirmation of prostate cancer, but the diagnostic accuracy of prostate biopsies is problematic. Treatment usually consists of radical prostatectomy, external beam irradiation, brachytherapy (interstitial implantation of radioactive seeds), androgen ablation (hormone) therapy, or "watchful waiting". In some cases, radiation therapy is prescribed to the pelvis and/or prostate bed even without confirmation by biopsy or imaging [5-7], despite the fact that irradiating a wide field in the pelvis (when disease is only suspected to extend beyond the known cancerous prostate) can lead to a higher incidence of post-irradiation morbidity than with irradiation of the prostate bed alone [8]. A major problem with prostatic cancer therapy is the question of when to treat or whether to treat at all. This is particularly problematic in the case of an increased PSA level after a prostatectomy. A new imaging technology for sensitive detection of early stage prostate cancer is needed to confirm PSA and help guide treatment decisions. In addition, a new method is needed to assess response shortly after treatment intervention.

In order to help meet these needs, we are designing a compact PET camera optimized to image the prostate. Functional PET imaging will help detect malignant tumors in the prostate and/or prostate bed to confirm an increased PSA level, as well as possibly help determine tumor "aggressiveness" based on metabolic uptake levels, in order to help guide whether to treat suspected prostatic cancer. Although not optimized to detect distant metastatic disease, this compact PET camera should also image local spread beyond the prostate bed to help guide treatment decisions such as whether a narrow or wide irradiation treatment field is needed.

Many groups have shown that PET imaging with $\left[{ }^{18} \mathrm{~F}\right]$ fluorodeoxyglucose (FDG) is not a good technique for prostate cancer diagnosis, because FDG is not very prostate specific (with a standardized uptake value of typically 2 ) primarily due to bladder accumulation. However, more promising PET radiopharmaceuticals have recently demonstrated outstanding results in the sensitive detection of prostate cancer, inspiring a new interest in using PET for prostate cancer imaging. Consistent with the evidence of increased pool size of choline in prostate cancer [9, 10], Hara and co-workers demonstrated prostate tumor uptake by $\left[{ }^{11} \mathrm{C}\right]$ choline. They find that: $\left[{ }^{11} \mathrm{C}\right]$ choline clears the blood faster than FDG; its uptake in prostate tumors is significantly higher than in normal and surrounding tissues [11, 12], 

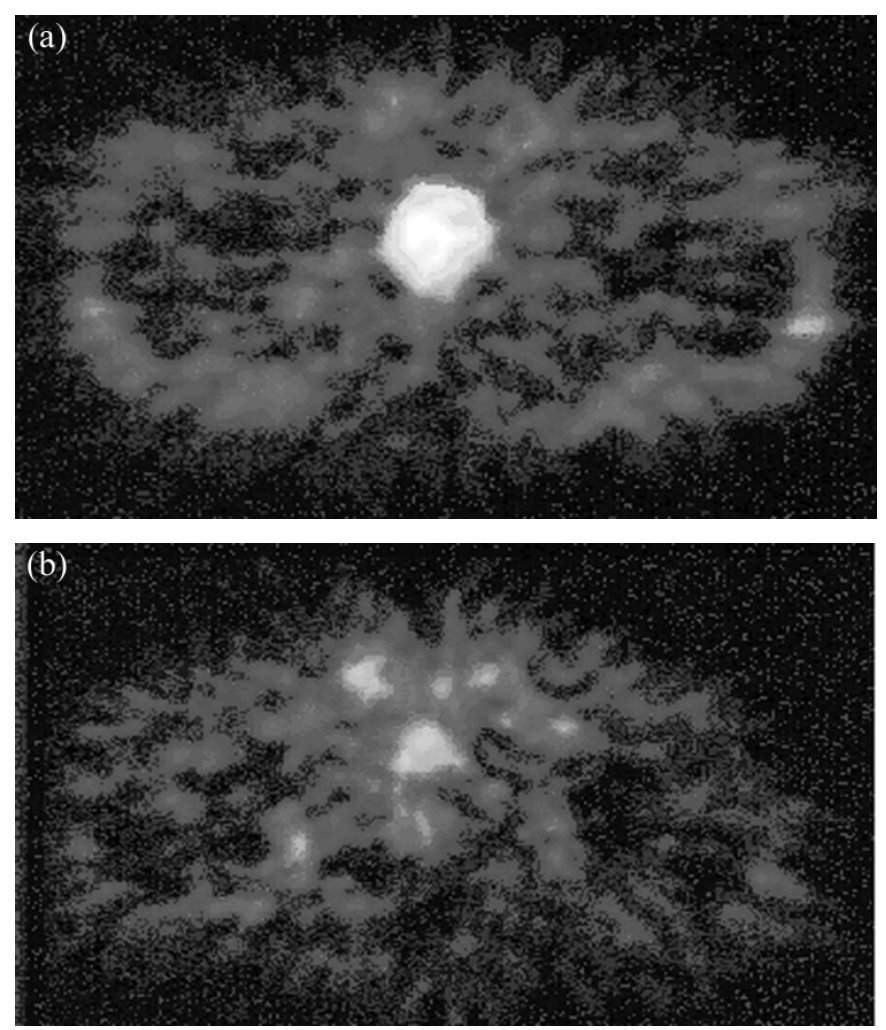

Fig. 1: $\left[{ }^{11} \mathrm{C}\right]$ choline image of prostate cancer (a) before and (b) after treatment. These grayscale images indicate a high (white) uptake in the prostate cancer compared with a low (gray) uptake elsewhere. Images are provided by Hara and co-workers [13].

providing excellent tumor/normal contrast; and bladder accumulation is minimal if the correct time course is chosen [13] which is a major advantage over FDG. Additionally, they observe that $\left[{ }^{11} \mathrm{C}\right]$ choline PET is more sensitive for detecting bone metastases than bone scintigraphy. Therefore, $\left[{ }^{11} \mathrm{C}\right]$ choline is an attractive PET tracer for imaging primary and metastatic tumors of the prostate and potentially for other regions of the body [14-18]. Figure 1 shows a $\left[{ }^{11} \mathrm{C}\right]$ choline image of prostate cancer before and after therapy, demonstrating the ability to detect prostate carcinoma using choline and possibly its analogs. Other ${ }^{11} \mathrm{C}$ radiopharmaceuticals are also under investigation for prostate cancer PET imaging, including $\left[{ }^{11} \mathrm{C}\right]$ acetate and $\left[{ }^{11} \mathrm{C}\right]$ methionine.

${ }^{18} \mathrm{~F}$ imaging has the advantage of a longer half-life, increasing commercial viability since an on-site cyclotron facility would not be necessary (as it would for ${ }^{11} \mathrm{C}$ imaging). There are several ${ }^{18} \mathrm{~F}$ radiopharmaceuticals currently under investigation for prostate cancer imaging, including $\left[{ }^{18}\right.$ F]fluorocholine (FCH) $[19,20]$. PET images using $\left[{ }^{18} \mathrm{~F}\right]$ fluorocholine demonstrate high standardized uptake values (e.g., SUV of 8), indicating that $\mathrm{FCH}$ is well localized in the prostate cancer and can be imaged with good resolution if a short scan time ( $\sim 5$ minutes $)$ is used. Therefore, $\left[{ }^{18} \mathrm{~F}\right]$ fluorocholine is also an attractive PET tracer for imaging primary and metastatic tumors of the prostate. (a)

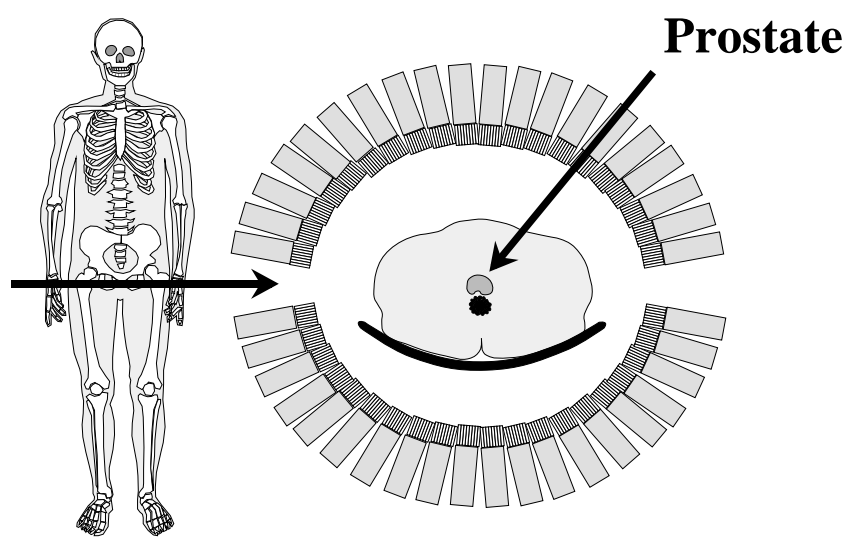

(b)

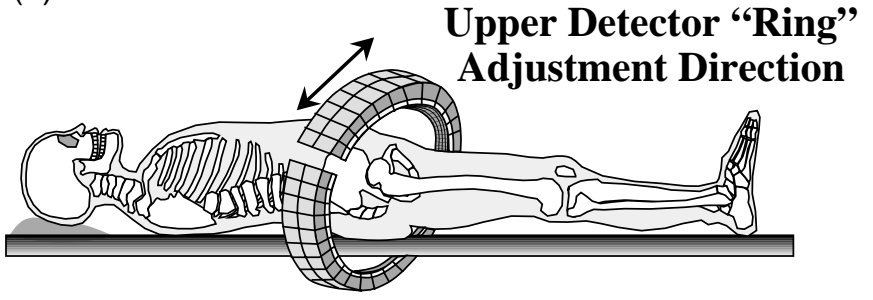

Fig. 2: Proposed positron tomograph for prostate imaging (shielding not shown). (a) Drawing of a transaxial view through prostate, showing the patient centered between two detector banks. The individual detector modules are angled to point towards the prostate. (b) Drawing of the sagittal view. The bottom arc is fixed below the patient bed, whereas the top arc adjusts vertically for patient access and compactness. Both detector banks are tilted and positioned as close as possible to the prostate, which improves sensitivity and minimizes attenuation.

\section{CAmera Design}

These new prostate tracers motivate us to design a low cost PET camera optimized to image the prostate. Coincidence imaging of positron emitters is achieved using a pair of external curved detector banks, one placed above and one below the patient. Fig. 2 shows the transaxial and sagittal views of the proposed camera design. The bottom bank is fixed below the patient bed, and the top bank moves upward for patient access and downward for maximum sensitivity. Each bank consists of 40 conventional block detectors such as those in the Siemens/CTI ECAT HR+ $(8 \mathrm{x}$ 8 array of $4.5 \mathrm{~mm} \times 4.5 \mathrm{~mm} \times 30 \mathrm{~mm}$ BGO crystals), forming two arcs with a minor axis of $44 \mathrm{~cm}$ and major axis of $60 \mathrm{~cm}$. The proposed prostate camera has one-half the axial coverage and uses about one-fourth the number of detectors as in a conventional PET system. However, since the average distance to the detectors is approximately one-half that of a conventional 2D PET system, the solid angle for a central source is approximately double.

Individual detector modules are angled to point towards the camera center near the prostate location in order to reduce penetration effects (in the detector) for annihilation photons originating in the prostate, which is an unique feature for a non-circular camera geometry. Annihilation photons from other parts of the field of view (FOV) will suffer increased 


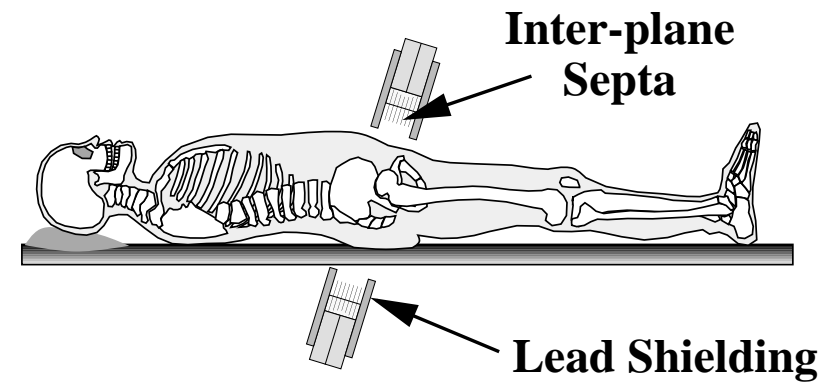

Fig. 3: Cut-out side view of the proposed positron tomograph, showing the shielding and inter-plane septa.

penetration effects, but these FOV regions are less important. Both detector banks are tilted to image the prostate while minimizing attenuation (i.e., above the buttocks and below the stomach), but the gantry can also allow zero tilt for thin patients. The patient is not fully encircled in 2D, which will be discussed further in Section III.

Standard block detectors with thick gamma ray detectors (i.e., 3 attenuation lengths) are needed for good detection efficiency. Narrow detector elements (i.e., $4.5 \times 4.5 \times 30 \mathrm{~mm}$ BGO crystals) are desired to achieve good spatial resolution. The $511 \mathrm{keV}$ photons of primary interest originate from the prostate which is roughly a small (e.g., $3 \mathrm{~cm}$ diameter) spherical source with a known central location at a reasonable distance from detector modules, so expensive detector modules with depth of interaction capability are not necessary. A two ring camera is more practical for patient positioning. Therefore, our proposed camera design attempts to maximize performance while minimizing cost, in order to produce a PET camera with nearly twice the detection efficiency as a conventional 2D PET camera with approximately one-quarter the detector costs.

The camera design also includes shielding, as shown in Fig. 3. Inter-plane septa extend $5 \mathrm{~cm}$ beyond the scintillator crystals to reduce background events from random

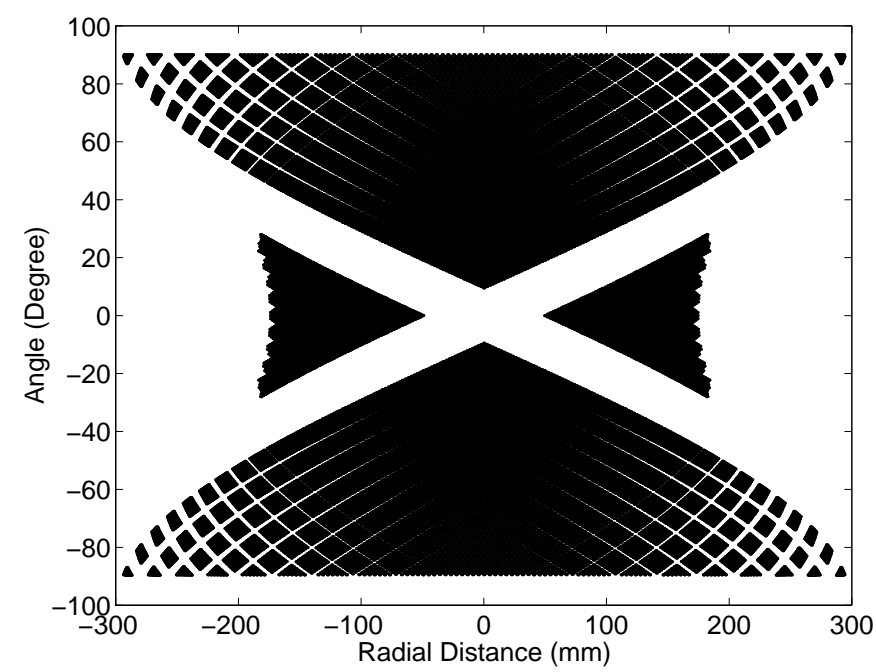

Fig. 4: Incomplete sampling for the field of view due to side gaps between detector banks. Coincidences between detector modules within the same bank and imaging plane are allowed, resulting in the $\sim$ triangular sampling regions on the left and right. The field of view of the proposed camera is an elliptical region, so the only missing samples are the " $\mathrm{X}$ " region in the center of the sinogram.

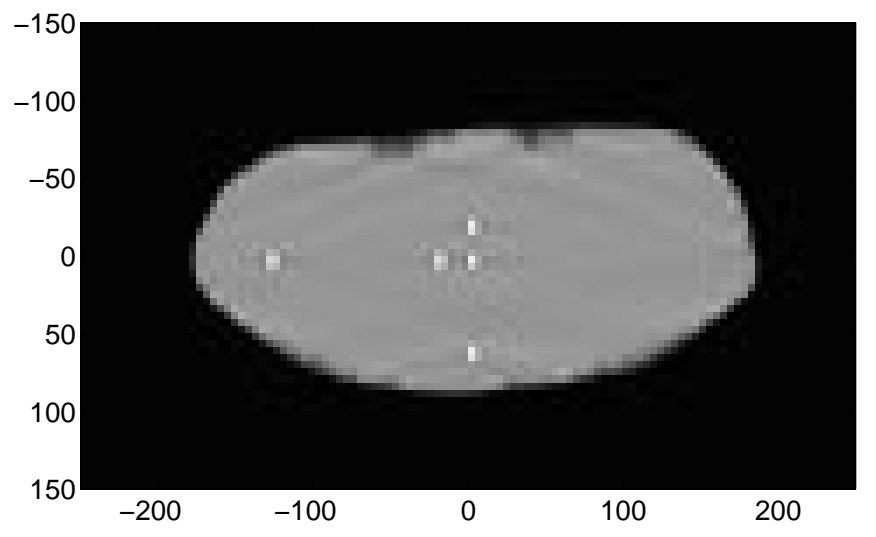

Fig. 5: Statistical noise-free reconstructed image of five point sources, demonstrating the local impulse response function and sampling sufficiency. Scale in millimeters.

coincidences and from photons that Compton scatter in the patient. Lead shields are also used on the ends to reduce activity from outside the field of view.

\section{EXPECTED PERFORMANCE}

\section{A. Simulation}

Our camera geometry provides a compact design (i.e., 44 $\mathrm{cm}$ minor axis between opposing detector faces) that adjusts vertically to fit the patient. However, a patient of average size will not be fully encircled by the detector rings, as mentioned in Section II. Fig. 4 shows the resulting irregular and incomplete sampling due to the side gaps. Similar irregular and incomplete sampling patterns have been dealt with in our positron emission mammography camera [21]. Despite this incomplete sampling, we are able to reconstruct nearly artifact free images in the region of interest by using an iterative reconstruction as described below. This iterative reconstruction algorithm can easily deal with the missing data of the system and can also partially recover the resolution loss (due to the crystal penetration effect) in the off-center FOV region. The gap size will increase for larger patients and this may impact the image quality, but we have not yet explored the effects of variations in gap size.

Our camera design includes inter-plane septa, so the data will be acquired in 2D. We use Monte Carlo data to simulate the camera performance. Reconstructions are performed with an iterative maximum likelihood algorithm [22]. Selfattenuation is simulated assuming a constant attenuation coefficient of $0.0095 / \mathrm{mm}$ inside the body, then attenuation is modeled in the reconstruction. Coincidences between detector modules within the same bank are allowed.

Fig. 5 shows an image of five point sources that are reconstructed without statistical noise to demonstrate how irregular sampling affects the local response function. All point sources are clearly visible. We observe increased radial blurring near the outer surface of the patient as expected due to penetration effects, but observe good resolution in the region near the center (i.e., near the prostate). In short, we are optimizing resolution at the center near the prostate at the price of resolution at the edges, which implies that distant 
metastases will suffer from some resolution degradation. Data are generated by forward projection of 256x256 $2 \mathrm{~mm} \times 2$ $\mathrm{mm}$ phantom voxels to simulate real data, and reconstruction is done with $128 \times 1284.51 \mathrm{~mm} \times 4.51 \mathrm{~mm}$ pixels, thus causing some point source spread among adjacent pixels.

As we are planning to use iterative methods to reconstruct images, the anticipated spatial resolution will depend on the geometrical parameters used for reconstruction (e.g., the voxel size and detector model) as well as the number of events. Thus, it is difficult to derive meaningful estimates of the expected spatial resolution from the test image in Fig. 5. Therefore, we estimate the spatial resolution analytically with the help of a simplifying assumption. That assumption is that if two tomographs employ the same scintillation material, the contribution of penetration to the spatial resolution for a source at any position within either camera depends only on the angle $\theta$ that the incoming gamma ray makes to the axis of the detector crystals (see Fig. 6). Given this assumption, we can use measured spatial resolutions [23] to estimate the resolution for the proposed camera. Fig. 6(b)

(a)

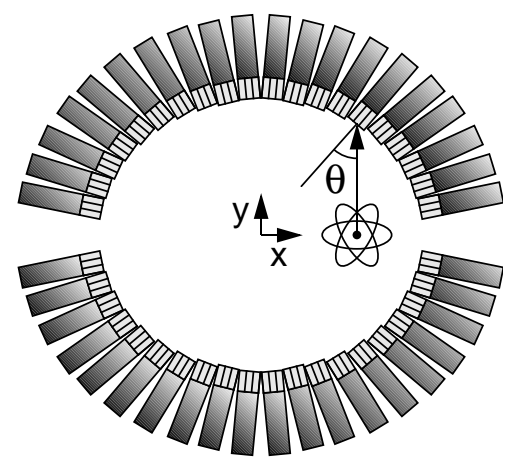

(b)

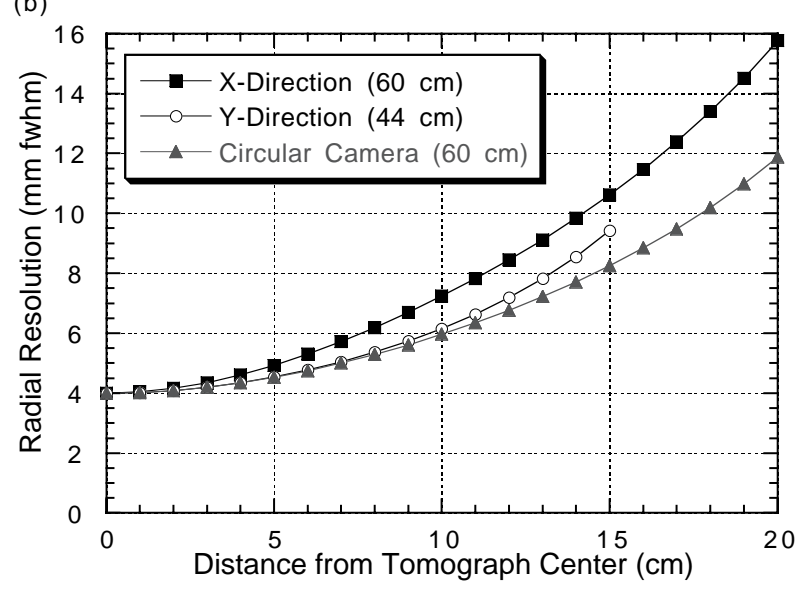

Fig. 6: (a) Proposed prostate PET camera drawing shown with a source within the camera. The angle $\theta$ is defined to be that which an incoming gamma ray makes to the axis of the detector crystal. (b) The anticipated spatial resolution for the proposed prostate PET camera as a function of distance from the center of the field of view along both the x- (square) and $y-$ (circle) axes, based on measured spatial resolutions with a circular tomograph. Little degradation due to penetration artifacts is seen for objects within $5 \mathrm{~cm}$ of the camera center, and $6 \mathrm{~mm}$ fwhm or better resolution is obtained within $8 \mathrm{~cm}$ of the camera center. Plot also shows spatial resolution for the reference circular PET camera as a function of distance from the center of the FOV along the $\mathrm{x}$ - (triangle) axis. shows the anticipated spatial resolution obtained in this manner as a function of distance from the center of the field of view (along both the $\mathrm{x}$ - and $\mathrm{y}$ - axes). Notice that little degradation due to penetration artifacts is seen for objects within $5 \mathrm{~cm}$ of the camera center, and that $6 \mathrm{~mm}$ fwhm or better resolution is obtained within $8 \mathrm{~cm}$ of the camera center. This region is easily large enough to contain the entire prostate.

We also use Monte Carlo data to simulate the performance of our proposed camera for imaging extended sources. Extended "prostate" sources are reconstructed with statistical noise representing 745 kcounts using a preliminary iterative maximum likelihood algorithm. We assume a $2.5 \mathrm{~cm}$ diameter spherical "prostate" (see fig. 7(a)) with a tumor to background ratio of $2: 1$, a $30 \%$ scatter fraction and $20 \%$ randoms fraction. The images are post-filtered with a Gaussian function having a standard deviation of 1 pixel. The spherical "prostate" is divided into quadrants. Fig. 7 (b) shows a reconstructed image with a tumor in the upper left quadrant of the spherical prostate. Fig. 7 (c) shows a reconstructed image with a tumor in the right half of the prostate. Fig 7 (d) shows a reconstructed image with a tumor in the upper right and lower left quadrants. These "prostate" tumors are clearly visible in all images, demonstrating that we can reconstruct nearly artifact free images near the prostate despite incomplete sampling and that the scanner can differentiate partial and whole prostate tumors. Our preliminary rate calculations (described below) indicate that 745 kcounts per imaging plane will be achievable with a 6minute scan after injecting a clinically acceptable $(10 \mathrm{mCi})$ patient dose of $\left[{ }^{11} \mathrm{C}\right]$ choline or $\left[{ }^{18} \mathrm{~F}\right]$ fluorocholine. A relatively short value of six minutes is chosen to demonstrate that good images can be obtained even if the bladder fills rapidly. All published $\left[{ }^{11} \mathrm{C}\right]$ choline or $\left[{ }^{18} \mathrm{~F}\right]$ fluorocholine studies done to date have used three minutes or longer (up to 30 minutes) imaging times.

\section{B. Estimated Rates}

The average distance between detector banks in our proposed camera is approximately one-half that of a conventional PET system such as the HR, so our sensitivity per slice is nearly 2 times higher than the HR (i.e., our detectors will have nearly twice the rate). However, our camera also has half the number of imaging planes, resulting in a total system rate that is approximately the same as the HR. Forty five minutes after injecting $15 \mathrm{mCi}$ of FDG into an average-sized patient (i.e., with $\sim 10 \mathrm{mCi}$ remaining in the patient), we see a $50 \mathrm{kHz}$ total coincidence rate using a $\mathrm{HR}$ scanner. Therefore, our total system coincidence rate is expected to be approximately $50 \mathrm{kHz}$, with a detector live time of at least $80 \%$.

For our proposed camera geometry, there are 166 total cross planes. Assuming a $50 \mathrm{kHz}$ total coincidence rate, this implies a rate of $300 \mathrm{~Hz}$ per cross plane. Summing over 7 cross plane slices per imaging plane, this corresponds to a 2 $\mathrm{kHz}$ coincidence rate per imaging plane. Therefore, 745 kcounts per imaging plane will be achievable with a 6-minute 

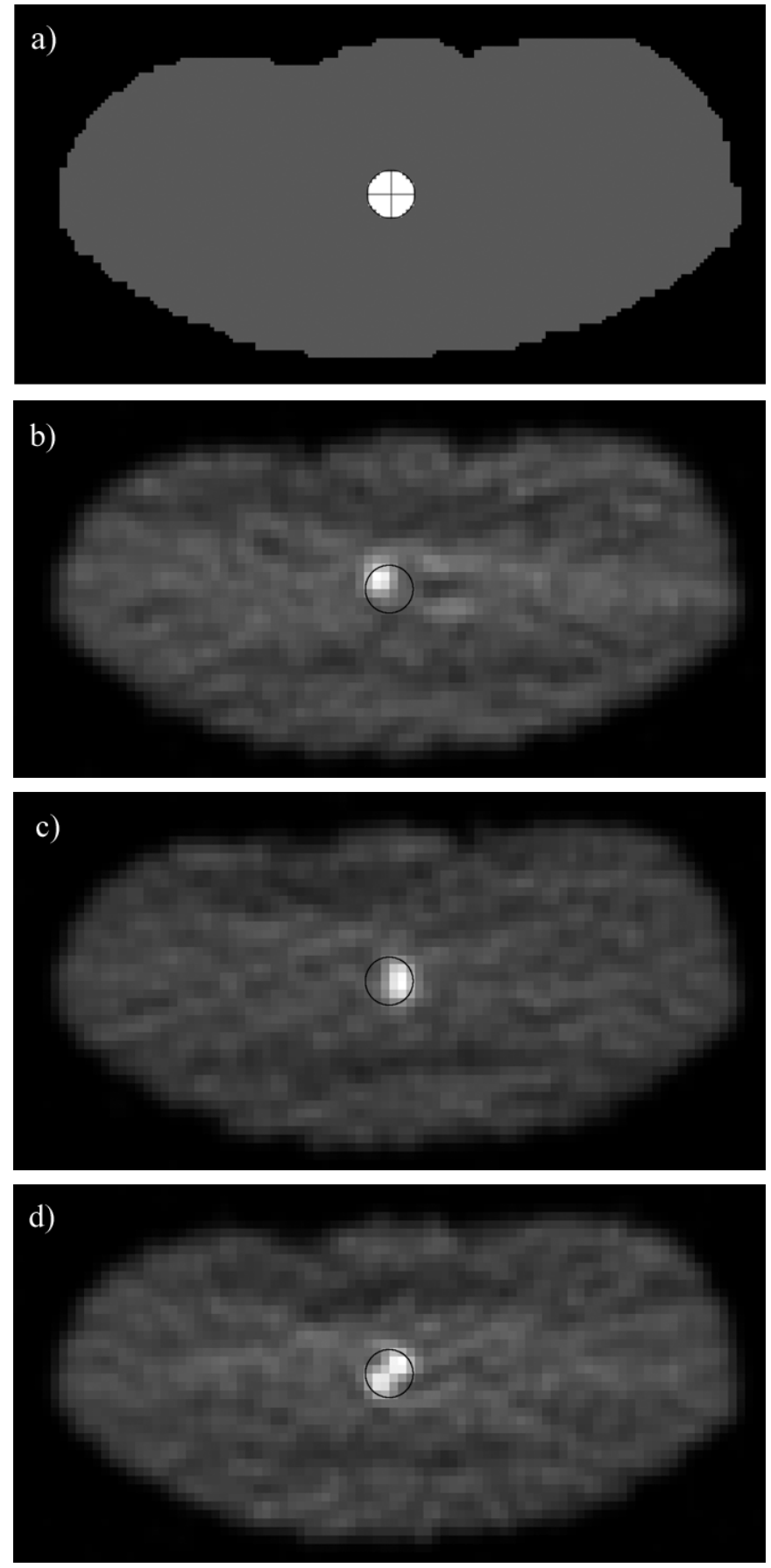

Fig. 7: Spherical "prostate" (2.5 cm diameter) is divided into quadrants, as shown in (a). Reconstructed image with a tumor in the (b) upper left quadrant, (c) right half, and (d) upper right and lower left quadrants of the prostate, assuming a tumor to background ratio of $2: 1,30 \%$ scatter fraction and $20 \%$ randoms fraction. All prostate tumors are clearly visible in all images, demonstrating that we can reconstruct nearly artifact free images near the prostate despite incomplete sampling and that the scanner can differentiate partial and whole prostate tumors.

scan after injecting a clinically acceptable $(10 \mathrm{mCi})$ patient dose of $\left[{ }^{11} \mathrm{C}\right]$ choline or $\left[{ }^{18} \mathrm{~F}\right]$ fluorocholine.

Rates are also computed using analytic estimates that were developed in our research group [24] and fit to published sensitivity and backgrounds of the HR tomograph [25]. They assume a $10 \mathrm{mCi}$ injection of $\left[{ }^{11} \mathrm{C}\right]$ choline or $\left[{ }^{18} \mathrm{~F}\right]$ fluorocholine uniformly distributed in a $70 \mathrm{kG}$ subject (torso $36 \mathrm{~cm}$ major axis, $17 \mathrm{~cm}$ minor axis), resulting in an activity concentration of $0.15 \mu \mathrm{Ci}$ per $\mathrm{ml}$. These calculated rates are in agreement with patient data within a factor of 2 .

\section{DATA CORRECTION}

This design does not include hardware for obtaining data to perform attenuation correction. The proposed design has sufficient room to incorporate a single photon or positron emitting source for transmission scans, but it would add to the camera cost. However, many clinical whole-body PET scans are done without an attenuation scan so it may not be necessary for the detection of abnormal activity in the prostate bed. A practical approach is to correct without additional hardware using calculated attenuation coefficients based on body contours (obtained from emission data) and an uniform attenuation coefficient. Anatomical boundaries are obtained from the outer edges of emission sinograms acquired from transverse sections [26]. This method can be generalized to three dimensions, and a uniform soft tissue attenuation coefficient can be used to compute the attenuation correction factors. This method assumes that the distribution of attenuating medium is uniform, that its external boundary is convex, that the attenuation coefficient for $511 \mathrm{keV}$ photons in soft tissue is uniform, and that inter-subject variability is small. These assumptions should be valid for the geometry and anatomy being imaged in this work. Attenuation can also be estimated using X-ray CT images to identify tissue types (bone, soft tissue, air, etc.), but this requires a second imaging procedure.

Because of the unusual geometry of our proposed camera, the sensitivity of each line of response depends on the distance between the two detectors; the shorter the distance is, the higher the sensitivity. Normalization will include geometric correction (sensitivity changes caused by the septa that are not modeled in the solid angle computation), block effect correction, individual detector sensitivity correction, and dead time correction. These factors will be obtained using a scan of an uniform activity cylindrical source such as used in conventional PET scanners. However, because of our specialized geometry and lack of rotational symmetry, the method for deriving the first two factors will be different from that of circular systems and may require a longer scan time to achieve good count statistics.

Random events will be estimated using the delayed window technique. Instead of pre-subtracting histogrammed data, delayed events will be saved in the list mode data stream with a tag to distinguish them from prompt events. Such information can be used to obtain a better estimate of the mean of the random events [27]. For our statistical reconstruction algorithm, the estimated randoms will be included in the forward model in reconstruction. Anatomical registration can be done using ultrasound or a point source.

\section{DISCUSSION}

This project is in its infancy, so there are many remaining uncertainties such as: which radiopharmaceutical will be used, the specific requirements for the radiopharmaceutical 
and imaging procedure, and the accuracy that physicians would require to perform effective diagnosis and evaluate response to therapy. However, new PET radiopharmaceuticals have recently demonstrated promising results in the sensitive detection of prostate cancer. Hence, a low cost PET scanner coupled with a ${ }^{18} \mathrm{~F}$-based isotope could make PET diagnosis of prostate cancer more clinically available. This PET camera design is also likely to out-perform whole body PET for this task, as its 2 times higher sensitivity will help for the short scans that appear to be necessary with choline-like radiopharmaceuticals due to bladder accumulation. We believe that providing improved PET imaging performance near the prostate bed at lower cost (than commercial PET) is clinically significant, outweighing the trade-off of some resolution degradation for distant metastases.

\section{CONCLUSION}

Promising new PET tracers for prostate cancer, such as $\left[{ }^{11} \mathrm{C}\right]$ choline and $\left[{ }^{18} \mathrm{~F}\right]$ fluorocholine, motivate us to build a low cost PET camera optimized for prostate imaging. Monte Carlo simulations demonstrate the feasibility of detecting and differentiating partial and whole prostate tumors with a tumor to background ratio of 2:1, using a 6-minute scan after a 10 $\mathrm{mCi}$ injection. Thus, the proposed camera will image prostate tumors with good spatial resolution and image contrast at low cost relative to commercial PET scanners.

\section{ACKNOWLEDGMENT}

We appreciate Dr. Hara from the International Medical Center of Japan for providing the PET images presented in Fig. 1 of this paper. This work was supported by the Director, Office of Science, Office of Biological and Environmental Research, Medical Science Division of the U.S. Department of Energy under contract No. DE-AC0376SF00098. Reference to a company or product name does not imply approval or recommendation by the University of California or the U.S. Department of Energy to the exclusion of others that may be suitable.

\section{REFERENCES}

[1] http://osp.nci.nih.gov/PRGReports/PPRGReport/toc.htm.

[2] H. I. Scher and L. W. Chung, "Bone metastases: improving the therapeutic index," Semin Oncol, vol. 21, pp. 630-56, 1994.

[3] H. I. Scher, M. Mazumdar, and W. K. Kelly, "Clinical trials in relapsed prostate cancer: defining the target," J Natl Cancer Inst, vol. 88, pp. 1623-34, 1996.

[4] M. A. Eisenberger and W. G. Nelson, "How much can we rely on the level of prostate-specific antigen as an end point for evaluation of clinical trials? A word of caution!," J Natl Cancer Inst, vol. 88, pp. 779-81, 1996.

[5] S. E. Schild, W. W. Wong, G. L. Grado, et al., "Radiotherapy for isolated increases in serum prostate-specific antigen levels after radical prostatectomy," Mayo Clin Proc, vol. 69, pp. 613-9, 1994.

[6] C. A. Perez and A. Eisbruch, "Role of post-radical prostatectomy irradiation in carcinoma of the prostate," Semin Rad Oncol, vol. 3, pp. 198, 1993.

[7] B. E. Shevlin, B. B. Mittal, W. N. Brand, et al., "The role of adjuvant irradiation following primary prostatectomy, based on histopathologic extent of tumor," Int J Rad Oncol Biol Phys, vol. 16, pp. 1425-30, 1989.

[8] J. S. Horoszewicz, E. Kawinski, and G. P. Murphy, "Monoclonal antibodies to a new antigenic marker in epithelial prostatic cells and serum of prostatic cancer patients," Anticancer Res, vol. 7, pp. 92735, 1987.

[9] J. Kurhanewicz, D. B. Vigneron, H. Hricak, et al., "Threedimensional $\mathrm{H}-1$ MR spectroscopic imaging of the in situ human prostate with high (0.24-0.7-cm3) spatial resolution," Radiology, vol. 198, pp. 795-805, 1996.

[10] J. Kurhanewicz, D. B. Vigneron, R. G. Males, et al., "The Prostate: Magnetic Resonance Imaging and Spectroscopy: Present and Future," in Radiologic Clinics of North America, H. Hricak and P. R. Carroll, Eds. New York, New York: W. B. Saunders Co., 2000, pp. 115-138.

[11] T. Hara, N. Kosaka, N. Shinoura, et al., "PET imaging of brain tumor with [methyl-C11]choline," J Nucl Med, vol. 38, pp. 842-7, 1997.

[12] N. Shinoura, M. Nishijima, T. Hara, et al., "Brain tumors: detection with C-11 choline PET," Radiology, vol. 202, pp. 497-503, 1997.

[13] T. Hara, N. Kosaka, and H. Kishi, "PET imaging of prostate cancer using carbon-11-choline," J Nucl Med, vol. 39, pp. 990-5, 1998.

[14] T. Hara, N. Kosaka, T. Kondo, et al., "Imaging of brain tumor, lung cancer, esophagus cancer, colon cancer, prostate cancer, and bladder cancer with [C-11]choline," J Nucl Med, vol. 38 (suppl), pp. 250P (abstract), 1997.

[15] T. Hara, K. Inagaki, N. Kosaka, et al., "Sensitive detection of mediastinal lymph node metastasis of lung cancer with 11C-choline PET," J Nucl Med, vol. 41, pp. 1507-13, 2000.

[16] I. J. Jong, T. H. Que, J. Pruim, et al., "Imaging of bladder cancer using carbon-11 choline positron emission tomography," J. Nucl. Med., vol. 41 (5 Suppl), pp. 74, 2000.

[17] J. Kotzerke, J. U. Prang, B. Neumaier, et al., "Carbon-11 choline positron emission tomography (PET) of prostate cancer -- first clinical experience," J. Nucl. Med., vol. 41 (5 Suppl), pp. 74, 2000.

[18] J. Kotzerke, J. Prang, B. Neumaier, et al., "Experience with carbon11 choline positron emission tomography in prostate carcinoma," Eur J Nucl Med, vol. 27, pp. 1415-9, 2000.

[19] T. R. Degrado, R. E. Coleman, S. W. Baldwin, et al., "Fluorine-18 fluorocholine $(\mathrm{FCH})$ as an oncological PET tracer: evaluation in murine prostate cancer xenograft model," J. Nucl. Med., vol. 41 (5 Suppl), pp. 231, 2000.

[20] T. R. Degrado, R. E. Coleman, S. Wang, et al., "Synthesis and Evaluation of F18-labeled Choline as an Oncologic Tracer for Positron Emission Tomography: Initial Findings in Prostate Cancer," Cancer Research, vol. 61(1), pp. 110-117, 2001.

[21] P. R. G. Virador, W. W. Moses, and R. H. Huesman, "Reconstruction in PET cameras with irregular sampling and depth of interaction capability," IEEE Trans Nucl Sci, vol. NS-45, pp. 1225-30, 1998.

[22] J. Qi, R. M. Leahy, S. R. Cherry, et al., "High-resolution 3D Bayesian image reconstruction using the microPET small-animal scanner," Phys Med Biol, vol. 43, pp. 1001-13, 1998.

[23] S. E. Derenzo, R. H. Huesman, J. L. Cahoon, et al., "Positron tomograph with 600 BGO crystals and $2.6 \mathrm{~mm}$ resolution," IEEE Trans Nucl Sci, vol. NS-35, pp. 659-64, 1988.

[24] S. E. Derenzo, "Method for optimizing side shielding in positron emission tomographs and for comparing detector materials," $J \mathrm{Nucl}$ Med, vol. 21, pp. 971-977, 1980.

[25] K. Wienhard, M. Dahlbom, L. Eriksson, et al., "The ECAT EXACT HR: performance of a new high resolution positron scanner," J Comp Asst Tom, vol. 18, pp. 110-118, 1994.

[26] T. F. Budinger and G. T. Gullberg, "Three-dimensional reconstruction in nuclear medicine emission imaging.," IEEE Trans Nucl Sci, vol. NS-21, pp. 2-20, 1974.

[27] E. Mumcuoglu, R. M. Leahy, and S. R. Cherry, "Bayesian reconstruction of PET images: methodology and performance analysis," Phys Med Bio, vol. 41, pp. 1777-1807, 1996. 